\title{
Genomic diversity of Ralstonia solanacearum strains isolated from banana farms in West Malaysia.
}

\begin{abstract}
Pulse-Field Gel Electrophoresis (PFGE) and BOX-PCR patterns were used to determine the phylogenetic relationships among 32 strains of Ralstonia solanacearum collected from banana farms in West Malaysia. Eighty two percent (25 stains) were biovar 3 and the remainders were biovar 4. Cluster analysis based on PFGE and BOX-PCR fingerprinting showed that there was significant genetic variation among all strains within this species in West Malaysia. Patterns of DNA fingerprinting in both methods were significantly correlated with sampling sites and revealed the regional similarity among the strains. The PFGE patterns varied from 78 to $99 \%$ while BOX-PCR patterns showed a high degree of variation among $\mathrm{R}$. solanacearum strains. Genomic fingerprinting by PFGE also revealed one that was significantly correlated to biovar type while BOX-PCR pattern analysis was unable to differentiate various biovars. This study clearly showed that $\mathrm{R}$. solanacearum strains were phylogenetically similar within a region but diverse between regions despite biovar designation.
\end{abstract}

Keyword: Ralstonia solanacearum; Genomic diversity; Banana diseases. 